\title{
Real-time collection of data on patients with COVID-19 and thrombosis and/or haemorrhage has aided the development of thromboembolism prophylaxis guidelines at the Royal Surrey County Hospital during the COVID-19 pandemic
}

\author{
Authors: Georgina Simpson ${ }^{\mathrm{A}}$ and Matthew Rogers ${ }^{\mathrm{A}}$
}

\section{Introduction}

As the COVID-19 pandemic progressed, it became apparent that worldwide these patients had a predisposition to thrombotic complications such as pulmonary embolisms, deep vein thromboses and circuit clotting in those receiving renal replacement therapy. ${ }^{1}$ At the start of the epidemic in the UK there was little trial evidence, and no national or international guidelines, to assist clinicians in optimising venous thromboembolism prevention and treatment for COVID-19 patients. It was therefore necessary to develop local guidelines, and to inform and fine-tune these we collected data on all patients with COVID-19 and thrombosis as the epidemic progressed.

\section{Method}

Patients were selected based on either having a positive swab or clinical/imaging features consistent with COVID-19 infection. From this cohort we identified those who had developed thromboembolism, confirmed on imaging, and/or patients who had suffered bleeding events. To identify these patients we liaised with various teams within the hospital including the intensive care unit, medicine and palliative care.

\section{Results and discussion}

We identified 25 patients based on our selection criteria. There were 21 thrombosis cases $(17=$ pulmonary emboli, $2=$ deep vein thrombosis, $2=$ other thrombosis) and 12 bleeding event cases $(2=$ minor (no intervention required), $9=$ clinically relevant but non-major (requiring some intervention but not lifethreatening), $1=$ major (life-threatening)).

Our study generated several key findings. The majority of thromboses were pulmonary emboli (17/21) and the majority of thromboses (13/21) occurred on or before admission and therefore patients were not receiving any anticoagulation at the

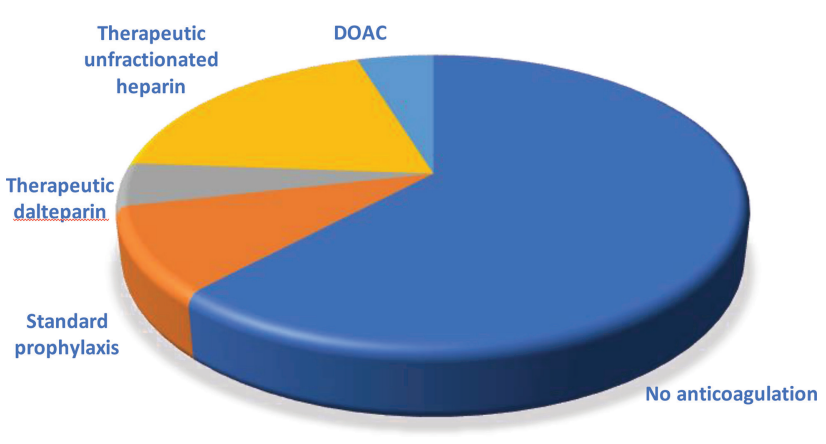

Fig 1. Anticoagulation being received at time of thrombosis diagnosis.

time (Fig 1). Not a single thrombosis occurred post-discharge, suggesting that post-discharge prophylaxis is not likely to be beneficial (Fig 2). This is in agreement with results from a larger study from King's College Hospital. ${ }^{2}$ Based on this finding, we removed the recommendation for routine post-discharge prophylaxis for COVID-19 patients from our guideline. There was a wide spread in D-dimer level (268-100,000 ng/mL), suggesting that D-dimer levels do not correlate well with

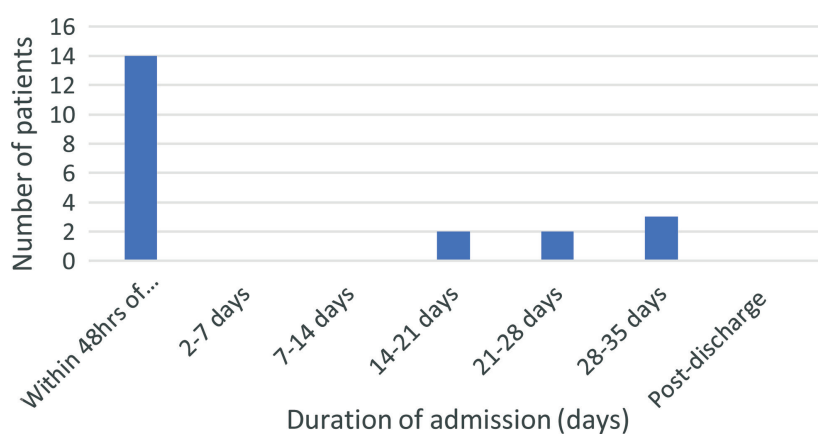

Fig 2. Time when thrombosis was diagnosed. 
thrombotic risk. We therefore removed 'high D-dimer' from our list of indications for considering escalated prophylactic dosing of low-molecular-weight heparin. The majority of bleeding events occurred while patients were on therapeutic unfractionated heparin (UFH). This may in part have been due to the fact these patients were the most unwell, but difficulties with monitoring UFH treatment in patients with COVID-19 may also have contributed: activated partial thromboplastin times may be misleading due to high levels of clotting factors in these patients. We have therefore moved to monitoring UFH treatment using anti-Xa levels.

\section{Conclusion}

By using real-time data collection during the course of the COVID-19 outbreak, we have been able to develop and refine our response in terms of our local venous thromboembolism guidelines. We are mindful of the dynamic situation surrounding the COVID-19 pandemic and plan to continue enhancing our guidelines as necessary.

\section{Conflicts of interest}

None declared.

\section{References}

1 Helms J, Tacquard C, Severac F et al. High risk of thrombosis in patients with severe SARS-CoV-2 infection: a multicenter prospective cohort study. Intensive Care Med 2020;46:1089-98.

2 Roberts LN, Whyte MB, Georgiou L et al. Postdischarge venous thromboembolism following hospital admission with COVID-19. Blood 2020;136:1347-50. 